The paper is presented at the conference "Complex analysis and its applications" (COMAN 2018), Gelendzhik - Krasnodar, Russia, June 2-9, 2018.

UDC $517.53 / .55$

A. B. SHISHKIN

\title{
SYMMETRIC REPRESENTATIONS OF HOLOMORPHIC FUNCTIONS
}

\begin{abstract}
.
In this article a class of symmetric functions is defined and used in some special representation of holomorphic functions. This representation plays an important role in transitions from concrete problems of projective description to equivalent problems of inductive description and finds multiple applications in questions connected with spectral synthesis of differential operators.
\end{abstract}

Key words: spectral synthesis, projective description, inductive description, differential operators, symmetric functions, holomorphic functions

2010 Mathematical Subject Classification: 32A10

1. Introduction. Suppose $n, q \in \mathbf{N} ; G$ is an open set in $\mathbf{C}^{n}$; $\pi: G \rightarrow \mathbf{C}^{q}$ is a holomorphic mapping. A set $g \subseteq G$ is called $\pi$-symmetric, if there exists a set $V$ in $\mathbf{C}^{q}$ such that $g=\pi^{-1}(V)$. A function $\varphi: g \rightarrow \mathbf{C}^{q}$, where $g$ is a $\pi$-symmetric set, is called $\pi$-symmetric on $g$, if $\varphi=\hat{\varphi} \circ \pi$, where $\hat{\varphi}$ is some holomorphic on $\pi(g)$ function. A set of all $\pi$-symmetric on $g$ functions $O_{\pi}(g)$ is a ring. This ring is a subring of the ring of all holomorphic on $g$ functions $O(g)$.

The class of $\pi$-symmetric functions is needed to consider some representations of holomorphic on complex domain functions. For example, consider the case of one variable. Suppose $n=q=1 ; \pi$ is a polynomial; $G$ is an open $\pi$-symmetric set in C. Note the following theorem: Any $u \in O(G)$ has the unique representation of the form $u(z)=\sum_{p=1}^{q-1} z^{p} u_{p}(z)$, where $u_{p} \in O_{\pi}(G)$ [1]. Such presentation is called a symmetric representation of the analytic function [2]. The case $\pi:=\left(\pi_{1}, \ldots, \pi_{q}\right): \mathbf{C} \rightarrow \mathbf{C}^{q}$,

(C) Petrozavodsk State University, 2018

\section{(cc) EY-NC}


where $\pi_{1}, \ldots, \pi_{q}$ are polynomials, was considered in [2]. The case where $G$ is an open set in $\mathbf{C},(G, \pi, \pi(G))$ - analytic covering, was studied in [3].

In this paper we consider a more general case. We obtain symmetric representations of some multivariate functions.

Note that the concept of symmetric representation of an analytic function plays a key role in some questions of complex analysis. For example it is used in spectral synthesis (see [3-6]).

\section{Analyticity of the difference relation.}

2.1. Alphabetized list of independent variables. Denote by $\tilde{\lambda}$ any product set $\left\{z_{1}^{(1)}, \ldots, z_{p(1)}^{(1)}\right\} \times \ldots \times\left\{z_{1}^{(n)}, \ldots, z_{p(n)}^{(n)}\right\}$, where $\left\{z_{1}^{(i)}, \ldots, z_{p(i)}^{(i)}\right\}$, $i=1, \ldots, n$ are ordered sets of independent variables.

The set $\tilde{\lambda}=\left\{z_{j}: j=1, \ldots, p\right\}$ has $p=p(1) \times \cdots \times p(n)$ different elements. Any finite sequence $z_{1}, \ldots, z_{p} \in \tilde{\lambda}$ is called an alphabetized list of the set $\tilde{\lambda}$, if $j<k \Leftrightarrow \exists m \in[1, n)$ such that $j_{1}=k_{1}, \ldots, j_{m}=k_{m}$, $j_{m+1}<k_{m+1}$ holds for any

$$
z_{j}=\left\{z_{j_{1}}^{(1)}, \ldots, z_{j_{n}}^{(n)}\right\}, z_{k}=\left\{z_{k_{1}}^{(1)}, \ldots, z_{k_{n}}^{(n)}\right\} .
$$

Let $z_{1}, \ldots, z_{p}$ be an alphabetized list of the set $\tilde{\lambda}$. Consider a matrix

$$
Z=\left(\begin{array}{ccc}
z_{1}^{(1)} & \ldots & z_{1}^{(n)} \\
z_{2}^{(1)} & \ldots & z_{2}^{(n)} \\
\ldots & \ldots & \ldots \\
z_{p(1)}^{(1)} & \ldots & z_{p(n)}^{(n)}
\end{array}\right)
$$

such that each $j$-th row equals to $z_{j}$. Choose any partition of the matrix $Z Z_{1}, \ldots, Z_{p(1)}$ where any submatrix $Z_{i}$ consists of $p(2) \cdots p(n)$ rows of the matrix $Z$. Partition of all $Z_{k}$ in the same way gives us submatrices $Z_{k, 1}, \ldots, Z_{k, p(2)}$, which consist of $p(3) \cdots p(n)$ rows of $Z$, etc. We have:

$$
Z=\left(\begin{array}{c}
Z_{1} \\
\vdots \\
Z_{p(1)}
\end{array}\right), Z_{k}=\left(\begin{array}{c}
Z_{k, 1} \\
\vdots \\
Z_{k, p(2)}
\end{array}\right), Z_{k, t}=\left(\begin{array}{c}
Z_{k, t, 1} \\
\vdots \\
Z_{k, t, p(3)}
\end{array}\right), \ldots
$$

Any submatrix of rank $m$ has the form:

$$
Z_{m}^{Z_{m}^{k, \ldots, j}}=\left(\begin{array}{ccc|ccc}
z_{k}^{(1)} & \ldots & z_{j}^{(m)} & z_{1}^{(m+1)} & \ldots & z_{1}^{(n)} \\
\ldots & \ldots & \ldots & \ldots & \ldots & \ldots \\
z_{k}^{(1)} & \ldots & z_{j}^{(m)} & z_{p(m+1)}^{(m+1)} & \ldots & z_{p(n)}^{(n)}
\end{array}\right),
$$


thus, columns $1, \ldots, m$ are equal.

Any two neighboring submatrices of rank $m$ are called adjacent if these submatrices are in the same submatrix of rank $m-1$ (note that $Z$ has rank 0). We have:

i) any two adjacent submatrices have the same columns except exactly one called marked;

ii) any marked column consists of equal elements.

More precisely, $m$-th columns of matrices $Z_{k, \ldots, j-1}$ and $Z_{k, \ldots, j}$ of rank $m$ are marked. The elements of $m$-th columns are equal to $z_{j-1}^{(m)}$ and $z_{j}^{(m)}$, respectively.

2.2. The main procedure. Let us consider the procedure used in the proof of theorem 1. Add the marked column of the upper adjacent submatrix to each submatrix $Z_{2}, \ldots, Z_{p(1)}$ of rank 1 from the right to obtain the non-rectangular matrix $Z^{\prime}$. Each submatrix $Z_{k, \ldots, j}$ of rank $m$ of matrix $Z$ determines the submatrix $Z_{k, \ldots, j}^{\prime}$ of rank $m$ of matrix $Z^{\prime}$ uniquely. Note that i) and ii) hold for adjacent pairs of submatrices of matrix $Z^{\prime}$. The pair $Z_{1}^{\prime}=Z_{1}, Z_{2}^{\prime}$ is the only exception. The marked columns of the adjacent matrix pair $Z_{k-1}^{\prime}$ and $Z_{k}^{\prime}, k=3, \ldots, p(1)$, are the last column of $Z_{k-1}^{\prime}$ and the first column of $Z_{k}^{\prime}$. Elements of these columns are equal to elements of $z_{k-2}^{(1)}$ and $z_{k}^{(1)}$, respectively. Add the marked column of upper adjacent submatrix to each submatrix $Z_{3}^{\prime}, \ldots, Z_{p(1)}^{\prime}$ of rank 1 from the right. We obtain a new matrix $Z^{\prime \prime}$ and new submatrices $Z_{k, \ldots, j}^{\prime \prime}$. Properties i) and ii) hold for $Z_{k, \ldots, j}^{\prime \prime}$. The pair $Z_{1}^{\prime \prime}=Z_{1}^{\prime}=Z_{1}, Z_{2}^{\prime \prime}=Z_{2}^{\prime}$ and $Z_{2}^{\prime \prime}=Z_{2}^{\prime}, Z_{3}^{\prime \prime}$ are exceptions. The marked columns of the adjacent matrix pair $Z_{k-1}^{\prime \prime}$ and $Z_{k}^{\prime \prime}, k=4, \ldots, p(1)$, are the last column of $Z_{k-1}^{\prime \prime}$ and the first column of $Z_{k}^{\prime \prime}$. Elements of these columns are equal to elements of $z_{k-3}^{(1)}$ and $z_{k}^{(1)}$, respectively. Then add columns to $Z_{4}^{\prime \prime}, \ldots, Z_{p(1)}^{\prime \prime}$, etc. Finely, $p(1)-1$-th step gives us the matrix ${ }_{1} Z$. We have:

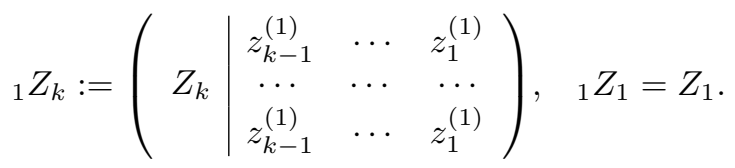

In the same way we deal with submatrices ${ }_{1} Z_{k}(\operatorname{rank}=1)$ of matrix ${ }_{1} Z$. Let us consider the first step. Properties i) and ii) are satisfied for adjacent submatrix pairs of matrix ${ }_{1} Z$, if the submatrices have rank $>1$. Then add the marked column of the upper adjacent submatrix to each 
submatrix ${ }_{1} Z_{k, 2}, \ldots,{ }_{1} Z_{k, p(2)}, k \in 1, \ldots, p(1)$ from the right to obtain a new matrix ${ }_{1} Z^{\prime}$ and new submatrices ${ }_{1} Z_{k, \ldots, j}^{\prime}$. Properties i) and ii) are satisfied for submatrices of rank $>2$. The marked columns of the adjacent matrix pair ${ }_{1} Z_{k, j-1}^{\prime},{ }_{1} Z_{k, j}^{\prime}, k=1, \ldots, p(1), j=3, \ldots, p(2)$, are the last column of ${ }_{1} Z_{k, j-1}^{\prime}$ and the second column of ${ }_{1} Z_{k, j}^{\prime}$. Elements of these columns are equal to elements of $z_{j-2}^{(2)}, z_{j}^{(2)}$ respectively. Then add columns to ${ }_{1} Z_{k, 3}^{\prime}, \ldots,{ }_{1} Z_{k, p(2)}^{\prime}$, etc. Finally, $p(2)-1$-th step gives us the matrix ${ }_{2} Z$. We obtain:

$$
\begin{aligned}
& { }_{2} Z_{k, j}=\left(\begin{array}{c|ccc|ccc}
Z_{k, j} & z_{k-1}^{(1)} & \cdots & z_{1}^{(1)} & z_{j-1}^{(2)} & \cdots & z_{1}^{(2)} \\
\cdots & \cdots & \cdots & \cdots & \cdots & \cdots \\
& z_{k-1}^{(1)} & \cdots & z_{1}^{(1)} & z_{j-1}^{(2)} & \cdots & z_{1}^{(2)}
\end{array}\right), \quad{ }_{2} Z_{1,1}=Z_{1,1},
\end{aligned}
$$

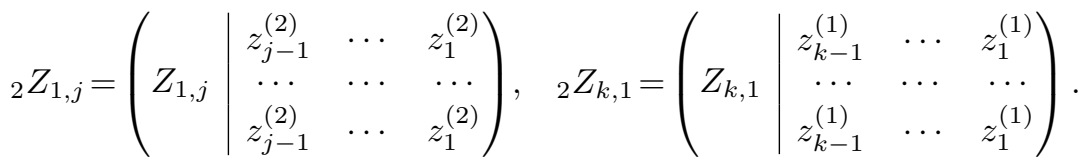

Then we change in the same way submatrices of ${ }_{2} Z$ of rank 2 , etc. We have:

$$
{ }_{n} Z_{k, \ldots, j}=\left(Z_{k, \ldots, j}\left|z_{k-1}^{(1)} \ldots z_{1}^{(1)}\right| \ldots \mid z_{j-1}^{(n)} \ldots z_{1}^{(n)}\right)
$$

(if $i \in k, \ldots, j$ and $i=1$, then there is no $i$-th submatrix in the matrix $\left.{ }_{n} Z_{k, \ldots, j}\right)$. Now we stop because submatrices of ${ }_{n} Z$ are rows of ${ }_{n} Z$ if these submatrices have rank $n$.

2.3. An analytic continuation of the difference relation. Choose any $m \times m$ invertible matrix $A=\left(a_{k, j}\right)$ and $l \times l$ nondegenerate matrix $B$. The matrix

$$
A \times B=\left(\begin{array}{ccc}
a_{11} B & \ldots & a_{1 m} B \\
\vdots & \ddots & \vdots \\
a_{m 1} B & \ldots & a_{m m} B
\end{array}\right) .
$$

is called the Kronecker product.

It is clear that the determinant $|A \times B|$ of the matrix $A \times B$ equals $|A|^{l}|B|^{m}$.

Suppose that $a_{1}, \ldots, a_{m} \in \mathbf{C}$. Consider the square matrix

$$
D\left(a_{1}, \ldots, a_{m}\right)=\left(\begin{array}{cccc}
1 & a_{1} & \ldots & a_{1}^{m-1} \\
\vdots & \vdots & \ddots & \vdots \\
1 & a_{m} & \ldots & a_{m}^{m-1}
\end{array}\right) .
$$


Determinant of the matrix $D\left(a_{1}, \ldots, a_{m}\right)$ is called the Vandermonde determinant. We have:

$$
\Delta\left(a_{1}, \ldots, a_{m}\right)=\prod_{1 \leqslant k \leqslant j \leqslant m}\left(a_{j}-a_{k}\right) .
$$

Let $\left\{z_{1}^{(i)}, \ldots, z_{p(i)}^{(i)}\right\}, i=1, \ldots, n$, be a collection of finite ordered number sets, $z_{1}, \ldots, z_{p}, p=p(1) \times \cdots \times p(n)$ is an alphabetized list of product set of sets $\left\{z_{1}^{(i)}, \ldots, z_{p(i)}^{(i)}\right\}, i=1, \ldots, n$. Consider the matrix

$$
D=D\left(z_{1}^{(1)}, \ldots, z_{p(1)}^{(1)}\right) \times \ldots \times D\left(z_{1}^{(n)}, \ldots, z_{p(n)}^{(n)}\right) ; \quad \Delta=\operatorname{det}(D) .
$$

It is clear that

$$
\Delta=\Delta_{1}^{p_{1}} \Delta_{2}^{p_{2}} \cdots \Delta_{n}^{p_{n}}=\prod_{i=1}^{n} \prod_{1 \leqslant k \leqslant j \leqslant m}\left(z_{j}^{(i)}-z_{k}^{(i)}\right)^{p_{i}},
$$

where $p_{i}=\frac{p}{p(i)}, \Delta_{i}=\Delta\left(z_{1}^{(i)}, \ldots, z_{p(i)}^{(i)}\right)$. We have:

$$
\Delta=\Delta\left(z_{1}, \ldots, z_{p}\right)=\left|\begin{array}{ccc}
z_{1}^{\alpha_{1}} & \ldots & z_{1}^{\alpha_{p}} \\
\vdots & \ddots & \vdots \\
z_{p}^{\alpha_{1}} & \ldots & z_{p}^{\alpha_{p}}
\end{array}\right|
$$

where $\alpha_{1}, \ldots, \alpha_{p}$ is an alphabetized list of the set

$$
\begin{gathered}
\{0, \ldots, p(1)-1\} \times \ldots \times\{0, \ldots, p(n)-1\} ; \\
\alpha_{k}=\left(\alpha_{k}^{(1)}, \ldots, \alpha_{k}^{(n)}\right) ; z_{j}^{\alpha_{k}}=\left(z_{j}^{(1)}\right)^{\alpha_{k}^{(1)}} \times \ldots \times\left(z_{j}^{(n)}\right)^{\alpha_{k}^{(n)}} .
\end{gathered}
$$

Let $g^{(1)}, \ldots, g^{(n)}$ be a collection of open sets in $\mathbf{C}$. Take the set

$$
\varphi_{1}\left(z^{(1)}, \ldots, z^{(n)}\right), \ldots, \varphi_{p}\left(z^{(1)}, \ldots, z^{(n)}\right)
$$

of functions that are holomorphic at the product set $g^{(1)} \times \ldots \times g^{(n)}$. Let

$$
\left\{z_{1}^{(1)}, \ldots, z_{p(1)}^{(1)}\right\}, \ldots,\left\{z_{1}^{(n)}, \ldots, z_{p(n)}^{(n)}\right\}
$$


be sets of independent variables where $z_{i}^{j} \in g^{(j)}$. Consider the relation

$$
F=\frac{|\Phi|}{\Delta}
$$

where $|\Phi|=\operatorname{det} \Phi$,

$$
\Phi=\left(\begin{array}{ccc}
\varphi_{1}\left(z_{1}\right) & \ldots & \varphi_{p}\left(z_{1}\right) \\
\vdots & \ddots & \vdots \\
\varphi_{1}\left(z_{p}\right) & \ldots & \varphi_{p}\left(z_{p}\right)
\end{array}\right)
$$

$z_{1}, \ldots, z_{p}$ is an alphabetized list of the product set

$$
\left\{z_{1}^{(1)}, \ldots, z_{p(1)}^{(1)}\right\} \times \ldots \times\left\{z_{1}^{(n)}, \ldots, z_{p(n)}^{(n)}\right\} .
$$

Determinants $|\Phi|$ and $\Delta$ are holomorphic functions on $g^{(i)}$. From Hartogs's theorem it follows that $|\Phi|$ and $\Delta$ are holomorphic at the product set

$$
\mathbf{g}=\underbrace{g^{(1)} \times \ldots \times g^{(1)}}_{p(1)} \times \ldots \times \underbrace{g^{(n)} \times \ldots \times g^{(n)}}_{p(n)} .
$$

Then $F$ is a holomorphic function at $\tilde{g} \backslash Z(\Delta)$, where $Z(\Delta)$ is a set of points of $\tilde{g}$ and $x \in Z(\Delta) \Rightarrow \Delta(x)=0$..

Theorem 1. The function $F$ has the unique holomorphic on $G$ analytical continuation.

Proof. It follows from continuity of $F$ that the analytical continuation is unique. It remains to check that it exists. Denote $\Phi$ by $\Phi(Z)$. Consider the partition of the matrix $\Phi(Z)$ :

$$
\Phi\left(Z_{k, 1}\right), \ldots, \Phi\left(Z_{k, p(2)}\right) .
$$

We have:

$$
\Phi(Z)=\left(\begin{array}{c}
\Phi\left(Z_{1}\right) \\
\vdots \\
\Phi\left(Z_{p(1)}\right)
\end{array}\right), \Phi\left(Z_{k}\right)=\left(\begin{array}{c}
\Phi\left(Z_{k, 1}\right) \\
\vdots \\
\Phi\left(Z_{k, p(2)}\right)
\end{array}\right), \ldots .
$$

If we replace $\Phi\left(Z_{k}\right), k=2, \ldots, p(1)$ by

$$
\Phi\left(Z^{\prime}\right)=\frac{\Phi\left(Z_{k}\right)-\Phi\left(Z_{k-1}\right)}{z_{k}^{(1)}-z_{k-1}^{(1)}},
$$


we obtain the matrix $\Phi\left(Z^{\prime}\right)$. Elements of $\Phi\left(Z^{\prime}\right)$ are holomorphic functions at g. We have:

$$
|\Phi(Z)|=\left(z_{p(1)}^{(1)}-z_{p(1)-1}^{(1)}\right)^{p_{1}} \times \ldots \times\left(z_{2}^{(1)}-z_{1}^{(1)}\right)^{p_{1}}\left|\Phi\left(Z^{\prime}\right)\right| .
$$

If we replace $\Phi\left(Z_{k}^{\prime}\right), k=3, \ldots, p(1)$ by

$$
\Phi\left(Z_{k}^{\prime \prime}\right)=\frac{\Phi\left(Z_{k}^{\prime}\right)-\Phi\left(Z_{k-1}^{\prime}\right)}{z_{k}^{(1)}-z_{k-1}^{(1)}},
$$

we get the matrix $\Phi\left(Z^{\prime \prime}\right)$. Elements of $\Phi\left(Z^{\prime \prime}\right)$ are holomorphic functions at $\mathbf{g}$. We have:

$$
\left|\Phi\left(Z^{\prime}\right)\right|=\left(z_{p(1)}^{(1)}-z_{p(1)-2}^{(1)}\right)^{p_{1}} \times \ldots \times\left(z_{3}^{(1)}-z_{1}^{(1)}\right)^{p_{1}}\left|\Phi\left(Z^{\prime \prime}\right)\right| .
$$

If we replace $\Phi\left(Z_{k}^{\prime \prime}\right), k=4, \ldots, p(1)$, etc, we get the matrix $\Phi\left({ }_{1} Z\right)$. Elements of $\Phi\left(Z^{\prime \prime}\right)$ are holomorphic functions at $\mathbf{g}$. We have

$$
|\Phi(Z)|=\Delta_{1}^{p_{1}}\left|\Phi\left({ }_{1} Z\right)\right| .
$$

Then we replace $\Phi\left({ }_{1} Z_{k, j}\right), j=2, \ldots, p(2)$ by

$$
\Phi\left({ }_{1} Z_{k, j}^{\prime}\right)=\frac{\Phi\left({ }_{1} Z_{k, j}\right)-\Phi\left({ }_{1} Z_{k, j-1}\right)}{z_{j}^{(2)}-z_{j-1}^{(2)}}
$$

in $\Phi\left({ }_{1} Z_{k}\right)$ for all $k \in 1, \ldots, p(1)$ to obtain the matrix $\Phi\left({ }_{1} Z^{\prime}\right)$. Its elements are holomorphic functions at $\mathbf{g}$. We have:

$$
\left|\Phi\left({ }_{1} Z\right)\right|=\left(z_{p(2)}^{(2)}-z_{p(2)-1}^{(2)}\right)^{p_{2}} \times \ldots \times\left(z_{2}^{(2)}-z_{1}^{(2)}\right)^{p_{2}}\left|\Phi\left({ }_{1} Z^{\prime}\right)\right| .
$$

If we replace $\Phi\left({ }_{1} Z_{k, j}^{\prime}\right), j=3, \ldots, p(2)$, etc, we get the matrix $\Phi\left({ }_{2} Z\right)$. Its elements are holomorphic functions at $\mathbf{g}$. We have:

$$
\left|\Phi\left({ }_{1} Z\right)\right|=\Delta_{2}^{p_{2}}\left|\Phi\left({ }_{2} Z\right)\right| \text {. }
$$

Then we get the matrix $\Phi\left({ }_{n} Z\right)$. Its elements are holomorphic functions at g. We have

$$
\left|\Phi\left({ }_{n-1} Z\right)\right|=\Delta_{n}^{p_{n}}\left|\Phi\left({ }_{n} Z\right)\right| .
$$

It follows from (1), (2), (3) that

$$
|\Phi(Z)|=\Delta\left|\Phi\left({ }_{n} Z\right)\right| .
$$


Finally, we obtain $F=\left|\Phi\left({ }_{n} Z\right)\right|$. This completes the proof.

\section{The symmetric representation of an analytic function.}

3.1. Analytic cover. Let $\Lambda$ be an image of $\pi: G \rightarrow \mathbf{C}^{q}$. The mapping $\pi$ is called an analytic cover of $\Lambda$ if the following conditions hold:

1) mapping $\pi$ is proper (hence, $\Lambda$ is an $n$-dimensional analytic set in $\mathbf{C}^{q}[4$, Remmert-Stein theorem $]$ );

2) there exists an analytic subset $\sigma \subset \Lambda, \operatorname{dim} \sigma<n$ such that $\Lambda_{*}=\Lambda \backslash \sigma$ is an n-dimensional complex manifold in $\mathbf{C}^{q}$;

3) the set $\pi^{-1}(\sigma)$ is nowhere dense in $G$;

4) the restriction of $\pi$ to $G_{*}=G \backslash \pi^{-1}(\sigma)$ is a local biholomorphic psheeted covering on $\Lambda_{*}$.

The set $\sigma$ is called critical. The preimage $\pi^{-1}(\sigma)$ is an $n$-dimensional subset of $G$. Metric dimension of $\pi^{-1}(\sigma)$ is less than or equal to $2 n-2$. Hence, $\pi^{-1}(\sigma)$ is removable. Then any bounded over $\pi^{-1}(\sigma)$ holomorphic on $G_{*}$ function has the unique holomorphic on $G$ extension. Sets $\pi^{-1}(\lambda)$, $\lambda \in \Lambda$ are compact analytic subsets of the set $G$ and are called $\pi$-layers. Hence, the sets are finite [7]. Points $\lambda \in \Lambda_{*}$ are called ordinary, and respective $\pi$-layers are called simple. Simple $\pi$-layers consist of $p$ different points. A single-valued mapping $\{1, \ldots, p\} \rightarrow \pi^{-1}(\lambda)$ is called ordering of a layer $\pi^{-1}(\lambda)$. Ordering of a simple $\pi$-layer can be represented in the form $z_{1}, \ldots, z_{p}$. Elements of the sequence $z_{1}, \ldots, z_{p}$ depend on $\lambda=\pi\left(z_{k}\right) \in \Lambda_{*}$. Mappings

$$
z_{k}=\left(z_{k}^{(1)}(\lambda), \ldots, z_{k}^{(n)}(\lambda)\right), k=1, \ldots, p,
$$

are holomorphic on some neighborhood of any ordinary point.

The concept of analytic cover develops the concept of local biholomorphic $k$-sheeted covering. Analytical coverings appear in the Weierstrass preparation theorem. It follows from the theorem that any pure $k$-dimensional analytical set has some analytical cover on $\mathbf{C}^{k}$ as a local representation.

3.2. Special analytic cover. Let $G^{(1)}, \ldots, G^{(n)}$ be open sets in $\mathbf{C} ; \pi^{(i)}: \mathbf{C} \rightarrow \mathbf{C}^{q(i)}, q(i) \in \mathbf{N}, i=1, \ldots, n$, be holomorphic functions; $\Lambda^{(i)}=\pi^{(i)}(\mathbf{C})$. The mappings $\pi^{(i)}$ are analytic covers, as:

1) the mapping $\pi^{(i)}: \mathbf{C} \rightarrow \mathbf{C}^{q(i)}$ is proper (hence, $\Lambda^{(i)}$ is 1-dimentional analytic set in $\mathbf{C}^{q(i)}$ ); 
2) there exists the close discrete set $\sigma^{(i)} \subset \Lambda^{(i)}$ such that $\Lambda_{*}^{(i)}=$ $\Lambda^{(i)} \backslash \sigma^{(i)}$ is a 1-dimentional complex manyfold in $\mathbf{C}^{q(i)}$;

3) the restriction of $\pi^{(i)}$ to $\mathbf{C}_{*}^{(i)}=\mathbf{C} \backslash\left(\pi^{(i)}\right)^{-1}\left(\sigma^{(i)}\right)$ is a local biholomorphic $p(i)$-sheeted covering on $\Lambda_{*}^{(i)}$.

It is clear that the product set $\tilde{\pi}: \mathbf{C}^{n} \rightarrow \mathbf{C}^{q}, q=q(1)+\ldots+q(n)$ of mappings $\pi^{(i)}, i=1, \ldots, n$, is an analytic covering. The image $\tilde{\pi}(\tilde{G})=\tilde{\Lambda}$ equals to product set $\Lambda^{(1)} \times \ldots \times \Lambda^{(n)}$ hence the image is an $n$-dimensional analytic set in $\mathbf{C}^{q}$ [7]. The mapping $\tilde{\pi}: \tilde{G} \rightarrow \tilde{\Lambda}$ is proper. Indeed, let $K$ be a compact set in $\tilde{\Lambda}$, let $K^{(i)}$ be a projection of $K$ on $\Lambda^{(i)}$, $\tilde{K}=K^{(1)} \times \ldots \times K^{(n)}$. It is clear that $\left(\pi^{(i)}\right)^{-1}\left(K^{(i)}\right)$ is a compact set in $G^{(i)}$.

$$
\tilde{\pi}^{-1}(\tilde{K})=\left(\pi^{(1)}\right)^{-1}\left(K^{(1)}\right) \times \ldots \times\left(\pi^{(n)}\right)^{-1}\left(K^{(n)}\right)
$$

$\tilde{\pi}^{-1}(\tilde{K})$ is a compact set in $\tilde{G} \cdot \tilde{\pi}^{-1}(K) \subseteq \tilde{\pi}^{-1}(\tilde{K})$ and $\tilde{\pi}^{-1}(K)$ are closed then $\tilde{\pi}^{-1}(K)$ is a compact set in $\tilde{G}$. This proves condition 1$)$. Further, critical set $\tilde{\sigma}$ equals to the set

$$
\tilde{\Lambda} \backslash\left(\Lambda_{*}^{(1)} \times \ldots \times \Lambda_{*}^{(n)}\right)=\bigcup_{i=1}^{n} \Sigma^{(i)},
$$

where

$$
\Sigma^{(i)}=\Lambda^{(1)} \times \ldots \times \Lambda^{(i-1)} \times \sigma^{(i)} \times \Lambda^{(i+1)} \times \ldots \times \Lambda^{(n)}
$$

are $(n-1)$-dimensional analytic subsets in $\tilde{\Lambda}$. Indeed, $\tilde{\sigma}$ is an $(n-1)$ dimensional analytic subset of $\tilde{\Lambda}$ and

$$
\tilde{\Lambda}_{*}=\tilde{\Lambda} \backslash \tilde{\sigma}=\Lambda_{*}^{(1)} \times \ldots \times \Lambda_{*}^{(n)}
$$

in an $n$-dimensional complex manifold in $\mathbf{C}^{q}$. We have proved that condition 2) holds. Set $\tilde{\pi}^{-1}(\tilde{\sigma})$ has the representation $\bigcup_{i=1}^{n} \tilde{\pi}^{-1}\left(\Sigma^{(i)}\right)$, where $\tilde{\pi}^{-1}\left(\Sigma^{(i)}\right)$ equals to

$$
G^{(1)} \times \ldots \times G^{(i-1)} \times\left(\pi^{(i)}\right)^{-1}\left(\sigma^{(i)}\right) \times G^{(i+1)} \times \ldots \times G^{(n)} .
$$

Since $\left(\pi^{(i)}\right)^{-1}\left(\sigma^{(i)}\right)$ is a closed discrete set, it follows that $\tilde{\pi}^{-1}\left(\Sigma^{(i)}\right)$ and $\tilde{\pi}^{-1}(\tilde{\sigma})$ are $(n-1)$-dimensional analytic sets. It now follows that $\tilde{\pi}^{-1}(\tilde{\sigma})$ is 
a nowhere dense set in $G$ [7]. Condition 3) holds. It is clear that condition $4)$ is satisfied. Note that $p=p(1) \ldots, p(n)$.

$\tilde{\Lambda}:=\Lambda^{(1)} \times \ldots \times \Lambda^{(n)}$ is a topological subspace of $\mathbf{C}^{q}$. Let $\Lambda$ be an open subset of $\tilde{\Lambda}, G=\pi^{-1}(\Lambda)$. A restriction $\pi: G \rightarrow \Lambda$ of the covering map $\tilde{\pi}: \mathbf{C}^{n} \rightarrow \mathbf{C}^{q}$ to the $\pi$-symmetric set $G$ is an analytic covering. We say that $\pi$ is special. Note that any $\pi$-symmetric set is $\tilde{\pi}$-symmetric. In particular, any $\pi$-layer equals to the respective $\tilde{\pi}$-layer.

3.3. Some representation of an analytic function. Let mapping $\pi: G \rightarrow \Lambda$ be a special analytic covering; let $\mathbf{O}(\Lambda)$ be a ring of holomorphic on $\Lambda$ functions; let $O\left(\Lambda_{*}\right)$ be a ring of locally holomorphic on $\Lambda_{*}$ functions; let $\mathbf{O}_{*}(\Lambda)$ be a subring of the ring $\mathbf{O}\left(\Lambda_{*}\right)$ that consists of bounded on $\Lambda$ functions. The mapping

$$
\mathbf{O}\left(\Lambda_{*}\right) \rightarrow \mathbf{O}_{\pi}\left(G_{*}\right) \mid \hat{\varphi} \rightarrow \hat{\varphi} \circ \pi
$$

is a ring isomorphism. Since the mapping $\pi$ is proper and the set $\pi^{-1}(\sigma)$ is removable, the restriction of the mapping $\pi$ to $\mathbf{O}_{*}(\Lambda)$ is a ring isomorphism. It takes $\mathbf{O}_{*}(\Lambda)$ on $\mathbf{O}_{\pi}\left(G_{*}\right) \cap O(G)$.

Let $\pi$ be a special analytic covering, $z \in G_{*}$ and

$$
\pi(z)=\lambda=\left(\lambda^{(1)}, \ldots, \lambda^{(n)}\right) \in \Lambda_{*}, \lambda^{(i)} \in \Lambda_{*}^{(i)} .
$$

Denote by $\tilde{\lambda}$ the $\pi$-layer $\pi^{-1}(\lambda)$. It is clear that $\tilde{\lambda}=\tilde{\lambda}^{(1)} \times \ldots \times \tilde{\lambda}^{(n)}$, where $\lambda^{(i)}$ are simple $\pi^{(i)}$-layers, $i=1, \ldots, n$. Since the $\pi$-layer $\tilde{\lambda}$ contains $z=\left(z^{(1)}, \ldots, z^{(n)}\right)$, then a $\pi^{(i)}$-layer $\tilde{\lambda}^{(i)}$ contains the $i$-th coordinate $z$. Let $z_{1}^{(i)}, \ldots, z_{p(i)}^{(i)}, z_{1}^{(i)}=z^{(i)}$, be an arbitrary ordering of a simple $\pi^{(i)}$-layer $\tilde{\lambda}^{(i)} ;$ let $z_{1}, \ldots, z_{p}$ be an alphabetized list of the layer $\tilde{\lambda}$. Consider a relation

$$
F=\frac{|\Phi|}{\Delta}
$$

where $|\Phi|=|\Phi|\left(z_{1}, \ldots, z_{p}\right), \Delta=\Delta\left(z_{1}, \ldots, z_{p}\right), \varphi_{1}, \ldots, \varphi_{p} \in O(G)$, define functions $f: G_{*} \rightarrow \mathbf{C}, \hat{f}: \Lambda_{*} \rightarrow \mathbf{C}$, with respect to the conditions

$$
f(z)=F\left(z_{1}, \ldots, z_{p}\right), \hat{f}(\lambda)=F\left(z_{1}(\lambda), \ldots, z_{p}(\lambda)\right) .
$$

Since the restriction of $F$ to $G \backslash Z(\Delta)$ is a symmetric function of variables $\left\{z_{1}^{(1)}, \ldots, z_{p(1)}^{(1)}\right\}, \ldots,\left\{z_{1}^{(n)}, \ldots, z_{p(n)}^{(n)}\right\}$ and $\left(z_{1}, \ldots, z_{p}\right) \in G \backslash Z(\Delta)$ for any $z \in G_{*}$, then the functions $f, \hat{f}$ are well defined. Indeed, the order of the set $\left\{z_{1}, \ldots, z_{p}\right\}$ changes but $F$ doesn not as one changes the 
order in a set $\left\{z_{1}^{(i)}, \ldots, z_{p(i)}^{(i)}\right\}, i \in 1, \ldots, n$. On the other hand, mappings $z_{1}(\lambda), \ldots, z_{p}(\lambda)$ are holomorphic on sufficently small neighborhoods of the ordinary points. Then $\hat{f} \in \mathbf{O}\left(\Lambda_{*}\right)$. It follows from theorem 1 that $\hat{f} \in \mathbf{O}_{*}(\Lambda)$. Indeed, suppose $\lambda=\left(\lambda^{(1)}, \ldots, \lambda^{(n)}\right) \in \sigma$ and let $k^{(i)} \subset \Lambda^{(i)}$ be a compact neighborhood of $\lambda^{(i)}$, let $d^{(i)} \subset \Lambda^{(i)}$ be an open neighborhood of compact $k^{(i)}, g^{(i)}=\left(\pi^{(i)}\right)^{-1}\left(d^{(i)}\right)$. Choose neighborhoods $k^{(i)}$, $d^{(i)}$ such that $\lambda^{(i)} \in \operatorname{int}\left(k^{(i)}\right) \neq \emptyset, \lambda \in d^{(1)} \times \ldots \times d^{(n)} \subseteq \Lambda, \tilde{\lambda} \subset$ $\subset g^{(1)} \times \ldots \times g^{(n)} \subseteq G$. Since the map $\pi^{(i)}$ is proper, $K^{(i)}=\left(\pi^{(i)}\right)^{-1}\left(k^{(i)}\right)$ is a compact set in $g^{(i)}$. It follows from theorem 1 that the function $F$ is analytic on a set $\mathbf{G}=\left(g^{(1)}\right)^{p(1)} \times \ldots \times\left(g^{(n)}\right)^{p(n)}$, hence, $F$ is bounded on $\left(K^{(1)}\right)^{p(1)} \times \ldots \times\left(K^{(n)}\right)^{p(n)}$. Then the function $\hat{f}$ is bounded on $k^{(1)} \times \ldots \times k^{(n)}$. Finally $\hat{f}$ is locally bounded on $\Lambda$.

We have

$$
f(z)=\hat{f}(\lambda)=(\hat{f} \circ \pi)(z),
$$

where $z \in G_{*}$ and $\lambda=\pi(z)$.

Now note that the function $f \in \mathbf{O}_{\pi}\left(G_{*}\right)$ is locally bounded on $\pi^{-1}(\sigma)$. The set $\pi^{-1}(\sigma)$ is removable. Then $f$ has an extension that is holomorphic on $G$. We have

$$
f \in \mathbf{O}_{\pi}\left(G_{*}\right) \cap O(G) .
$$

Now we can prove the following theorem.

Theorem 2. For any function $f \in O(G)$ the unique representation

$$
f=\sum_{k=1}^{p} z^{\alpha_{k}} f^{(k)}, f^{(k)} \in \mathbf{O}_{\pi}\left(G_{*}\right) \cap O(G) .
$$

is true. The restriction of $f^{(k)}$ to $G_{*}$ equals to $\Delta_{k}(f) / \Delta$, where $\Delta_{k}(f)$ is the determinant obtained by replacement of $k$-th column by the column from $f\left(z_{1}\right), \ldots, f\left(z_{p}\right)$ in determinant $\Delta$ and $z_{1}, \ldots, z_{p}$ is a alphabetized list of the simple $\pi$-layer $\tilde{\lambda}$ that contains $z$.

Proof. It is clear that for any $z \in G_{*}$ we have

$$
\tilde{f} \Delta=\sum_{k=1}^{p} \tilde{z}^{\alpha_{k}} \Delta_{k}(f),
$$


where $\tilde{f}=\left(f\left(z_{1}\right), \ldots, f\left(z_{p}\right)\right), \tilde{z}^{\alpha_{k}}=\left(z_{1}^{\alpha_{k}}, \ldots, z_{p}^{\alpha_{k}}\right)$. The determinant $\Delta$ is not equal to zero for any $z \in G_{*}$. Then for any $z \in G_{*}$ we have

$$
\tilde{f}=\sum_{k=1}^{p} \tilde{z}^{\alpha_{k}} f^{(k)},
$$

where $f^{(k)}=\Delta_{k}(f) / \Delta \in O_{\pi}\left(G_{*}\right)$. The functions $f^{(k)}$ have the only extension that is in $O(G)$. Now we have

$$
f^{(k)} \in \mathbf{O}_{\pi}\left(G_{*}\right) \cap O(G), k=1, \ldots, p .
$$

It is easy to see that $(2) \Leftrightarrow(5)$. Uniqueness of (2) follows from the fact that we obtain $f^{(k)}$ from (5), using Cramer's rule. Indeed,

$$
\left\{\begin{array}{l}
f\left(z_{1}\right)=z_{1}^{\alpha_{1}} f^{(1)}\left(z_{1}\right)+\ldots+z_{1}^{\alpha_{p}} f^{(p)}\left(z_{1}\right) \\
\cdot \cdot \cdot \\
f\left(z_{p}\right)=z_{p}^{\alpha_{1}} f^{(1)}\left(z_{p}\right)+\ldots+z_{p}^{\alpha_{p}} f^{(p)}\left(z_{p}\right)
\end{array}\right.
$$

The theorem is proved.

\section{References}

[1] Krasichkov-Ternovskii I. F. Spectral synthesis in a complex domain for a differential operator with constant coefficients. I: A duality theorem Mat. Sb., 1991, vol. 182, no. 2, pp. 1559-1587. DOI: https://doi.org/10. 1070/SM1993v074n02ABEH003349.

[2] Krasichkov-Ternovskii I. F. , Shishkin A. B. Local description of closed submodules of a special module of entire functions of exponential type. Mat. Sb., 2001, vol. 192, no. 11, pp. 1621-1638. DOI: https://doi.org/10. 4213/sm608.

[3] Shishkin A. B. Projective and injective descriptions in the complex domain. Duality. Izv. Saratov Univ. (N.S.), Ser. Math. Mech. Inform., 2014, vol. 14, no. 1 , pp. $47-65$.

[4] Shishkin A. B. Spectral synthesis for an operator generated by multiplication by a power of the independent variable. Mat. Sb., 1991, vol. 182, no. 6, pp. 828-848. DOI: https://doi.org/SM1992v073n01ABEH002542

[5] Shishkin A. B. Spectral synthesis for systems of differential operators with constant coefficients. Duality theorem. Mat. Sb., 1998, vol. 189, no. 9, pp. 143-160. DOI: https://doi.org/10.1070/ SM1998v189n09ABEH000355. 
[6] Shishkin A. B. Spectral synthesis for systems of differential operators with constant coefficients. Mat. Sb., 2003, vol. 194, no. 12, pp. 123-160. DOI: https://doi.org/10.1070/SM2003v194n12ABEH000789.

[7] Chirka E. M. Complex Analytic Sets. Kluwer Academic Publishers, 1989.

Received May 18, 2018.

In revised form, September 3, 2018.

Accepted September 3, 2018.

Published online September 18, 2018.

A. B. Shishkin

Kuban State University, branch in Slavyansk-on-Kuban

200, Kubanskaya str., Slavyansk-on-Kuban, Russia

E-mail: shishkin-home@mail.ru 